\title{
Nonequilibrium phase transitions induced by multiplicative noise
}

\author{
C. Van den Broeck \\ Limburgs Universitair Centrum, B-3590 Diepenbeek, Belgium \\ J. M. R. Parrondo \\ Departamento de Física Aplicada I, Universidad Complutense Madrid, E-28040 Madrid, Spain \\ R. Toral \\ Departament de Física, Universitat de les Illes Balears, and Instituto Mediterráneo de Estudios Avanzados, \\ IMEDEA (CSIC-UIB), E-07071 Palma de Mallorca, Spain \\ R. Kawai \\ Department of Physics, University of Alabama at Birmingham, Birmingham, Alabama 35294
}

(Received 15 July 1996)

\begin{abstract}
We review a mean-field analysis and give the details of a correlation function approach for spatially distributed systems subject to multiplicative noise, white in space and time. We confirm the existence of a pure noise-induced reentrant nonequilibrium phase transition in the model introduced in [C. Van den Broeck et al., Phys. Rev. Lett. 73, 3395 (1994)], give an intuitive explanation of its origin, and present extensive simulations in dimension $d=2$. The observed critical properties are compatible with those of the Ising universality class. [S1063-651X(97)08704-7]
\end{abstract}

PACS number(s): $05.40 .+\mathrm{j}$

\section{INTRODUCTION}

Noise is usually thought of as a phenomenon which perturbs the observation and creates disorder. This idea is based mainly on our day to day experience and, in the context of physical theories, on the study of equilibrium systems. The effect of noise can, however, be quite different in nonlinear nonequilibrium systems. Several situations have been documented in the literature, in which the noise actually participates in the creation of ordered states or is responsible for surprising phenomena through its interaction with the nonlinearities of the system [1-10]. Recently [11], a quite spectacular phenomenon was discovered in a specific model of a spatially distributed system with multiplicative noise, white in space and time. It was found that the noise generates an ordered symmetry-breaking state through a genuine secondorder phase transition, whereas no such transition is observed in the absence of noise. The purpose of this paper is to present a more detailed investigation of this phenomenon. First, we will give an intuitive explanation of why the transition occurs in this particular model and not in others. This explanation also sheds light on why phase transitions were not discovered in the related context of noise-induced transitions [1]. Second, after reviewing the mean-field analysis which was introduced in [12], we present the details of a more sophisticated approach, which involves the approximate calculation of the spatial correlation function. Third, we include extensive simulations of the model in spatial dimension $d=2$, and present a finite-size scaling analysis showing that the critical properties of the phase transition are compatible with those of the dynamical Landau-Ginzburg model or the Ising model.

\section{ZERO-DIMENSIONAL MODELS: SHORT-TIME VS LONG-TIME BEHAVIOR}

Consider the stochastic differential equation

$$
\dot{x}=f(x)+g(x) \xi
$$

where $\xi$ stands for Gaussian white noise with first two moments

$$
\begin{gathered}
\langle\xi(t)\rangle=0, \\
\left\langle\xi(t) \xi\left(t^{\prime}\right)\right\rangle=\sigma^{2} \delta\left(t-t^{\prime}\right) .
\end{gathered}
$$

Equation (1) is interpreted according to the Stratonovitch interpretation [13]. Hence the probability density $P(x, t)$ for the variable $x(t)$ obeys the Fokker-Planck equation $[1,14]$

$$
\partial_{t} P(x, t)=-\partial_{x}[f(x) P(x, t)]+\frac{\sigma^{2}}{2} \partial_{x}\left\{g(x) \partial_{x}[g(x) P(x, t)]\right\},
$$

and the steady-state solution is given by

$$
P^{\mathrm{st}}(x)=N \exp \left\{\int_{0}^{x} \frac{f(y)-\frac{\sigma^{2}}{2} g(y) g^{\prime}(y)}{\frac{\sigma^{2}}{2} g^{2}(y)} d y\right\},
$$

where $N$ is a normalization constant and $g^{\prime}(x)$ stands for the derivative of $g(x)$ with respect to its argument. The extrema $\bar{x}$ of the steady-state density obey the following equation:

$$
f(\bar{x})-\frac{\sigma^{2}}{2} g(\bar{x}) g^{\prime}(\bar{x})=0 .
$$


One notes that this equation is not identical to the equation $f(\bar{x})=0$ for the steady states in the absence of multiplicative noise. As a result, the most probable states need not coincide with the deterministic stationary states. More importantly, solutions can appear or existing solutions can be "destabilized"' by the noise. These changes in the asymptotic behavior of the system have been generally named noise-induced transitions [1].

To illustrate this phenomenon, consider the case of a deterministically stable steady state at $x=0$, e.g., $f(x)=-x+o(x)$, perturbed by a multiplicative noise. As is clear from Eqs. (4) and (5), a noise term of the form $g(x)=1+x^{2}+o\left(x^{2}\right)$ will have a stabilizing effect, since $-\left(\sigma^{2} / 2\right) g(\bar{x}) g^{\prime}(\bar{x})=-\sigma^{2} \bar{x}+o(\bar{x})$, and it makes the coefficient of $x$ more negative. On the other hand, noise of the form $g(x)=1-x^{2}+o\left(x^{2}\right)$, i.e., with maximal amplitude at the reference state $x=0$, has the tendency to "destabilize", the reference state. In fact, above a critical intensity $\sigma^{2}>\sigma_{c}^{2}=1$, the stationary probability density will no longer have a maximum at $\bar{x}=0$, and "noise-induced"' maxima can appear. This phenomenon remains possible even if the deterministic steady-state equation, obtained by fixing the random value of the noise to a constant value $\lambda$, namely, $f(\bar{x})+\lambda g(\bar{x})=0$, has a unique solution for all $\lambda$. Hongler's model [17], with $f(x)=-\tanh x=-x+o(x)$ and $g(x)=\operatorname{sech} x=1-x^{2} / 2+o\left(x^{2}\right)$, is a concrete example of this situation: for $\sigma^{2}>\sigma_{c}^{2}=2$, two noise-induced maxima arise on both sides of the deterministic reference state $\bar{x}=0$. One has coined the term "pure noise-induced transition" for this type of transitions.

Following the formalism for equilibrium states, it is tempting to introduce the notion of a "stochastic potential" $U_{\text {st }}(x)$ by writing: $P^{\text {st }}(x) \sim \exp \left[-U_{\text {st }}(x)\right]$. One concludes that for a system undergoing a noise-induced transition, e.g., for $g(x)=1-x^{2}+o\left(x^{2}\right)$, and for $\sigma^{2}>\sigma_{c}^{2}$, the stochastic potential has two minima. Consider now a spatially extended system obtained by coupling such units. The coupling is such that it favors the nearest-neighbor units, to stay at the same maximum of the probability density (minimum of the stochastic potential). In analogy to what happens for equilibrium models, such as the Landau-Ginzburg model $[18,19]$, one expects that this system will undergo a phase transition for some critical value of the "temperature", (noise intensity) $\sigma^{2}$. However, it turns out that this is not the case. In fact, we will show in the next sections that one needs a noise of precisely the other type, namely $g(x)=1+x^{2}+o\left(x^{2}\right)$, to generate a genuine phase transition. The reason for this counterintuitive result can be clarified by focusing on the shorttime behavior.

From Eq. (3), we obtain the following exact equation for the time evolution of the first moment of the probability density:

$$
\langle\dot{x}\rangle=\langle f(x)\rangle+\frac{\sigma^{2}}{2}\left\langle g(x) g^{\prime}(x)\right\rangle .
$$

When $f$ and/or $g$ are nonlinear, the evolution of the first moment is coupled to higher-order moments. Suppose, however, that we start with an initial Dirac $\delta$ probability density, and follow it for a short time, such that fluctuations are small and the probability density is well approximated by a Gauss-

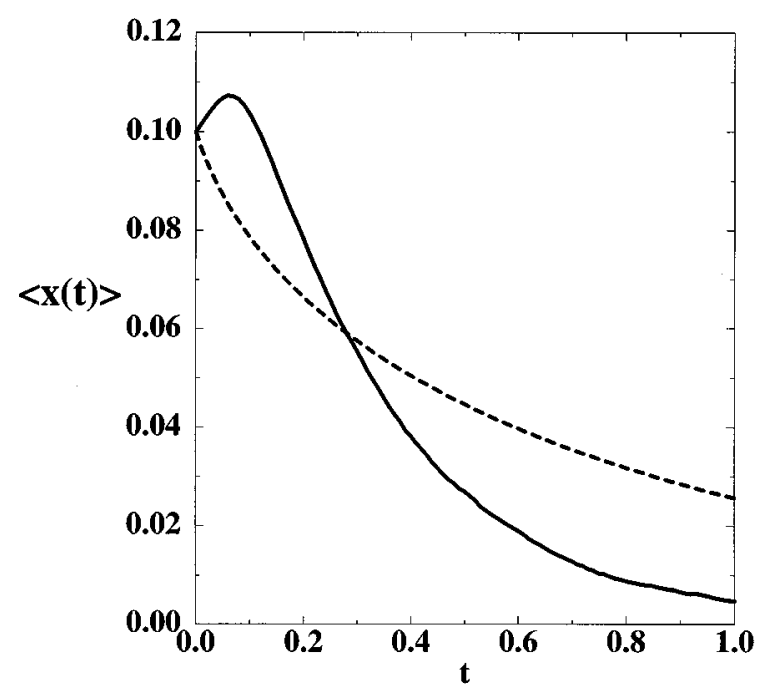

FIG. 1. Time-dependent evolution of the first moment $\langle x(t)\rangle$, starting from an initial state $P(x, t=0)=\delta(x-0.1)$, of a stochastic variable satisfying Eq. (1), for Hongler's model (dashed line), $f(x)=-\tanh x$ and $g(x) x=\operatorname{sech} x \approx 1-x^{2} / 2$, and for the model introduced in [11] (full line), $f(x)=-x\left(1+x^{2}\right)^{2}$ and $g(x)=1+x^{2}$. Notice that, for Hongler's model, the decay is monotonic, whereas for the other model there is a tendency to initially destabilize small values of $x$.

ian. The equation for the maximum of the probability, which is also the average value in this approximation $\bar{x}=\langle x\rangle$, takes on the following form [valid if $f(\langle x\rangle) \gg\left\langle\delta x^{2}\right\rangle f^{\prime \prime}(\langle x\rangle)$, and a similar condition for the term involving $g(x)]$ :

$$
\dot{\bar{x}}=f(\bar{x})+\frac{\sigma^{2}}{2} g(\bar{x}) g^{\prime}(\bar{x}) \text {. }
$$

The important observation to make is that the sign of the multiplicative noise term is opposite to that appearing in the long-time result, cf. Eq. (5). Hence it predicts an opposite effect of the multiplicative noise at early times. In particular, if we were to probe the "stability" of the reference state $\bar{x}=0$, we would conclude from Eq. (7) that a noise of the form $g(x)=1+x^{2}+o\left(x^{2}\right)$ now has the tendency to destabilize the reference state $\bar{x}=0$, favoring initially non-null values of the variable $x$.

To illustrate this point further, in Fig. 1 we have represented the time-dependent evolution of the first moment $\langle x(t)\rangle$, starting from an initial state $P(x, t=0)=\delta(x-0.1)$, for Hongler's model, $f(x)=-\tanh x$ and $g(x)=\operatorname{sech} x$, and for the model introduced in Ref. [11], $f(x)=-x\left(1+x^{2}\right)^{2}$ and $g(x)=1+x^{2}$. For Hongler's model, the analytic result is available. For the other model, $\langle x(t)\rangle$ was obtained through a numerical integration of the corresponding Langevin equation. If one would like to interpret these results again in terms of an equilibrium picture with a Brownian particle in an effective potential $U_{\text {eff }}(x)$, one finds that the short-time behavior corresponds to an effective potential with a single minimum at $x=0$ for Hongler's model, while it it bistable for the other model. In other words, the picture is just the reverse of the one suggested by the consideration of the steady-state probability and the stochastic potential $U_{\mathrm{st}}(x)$. 

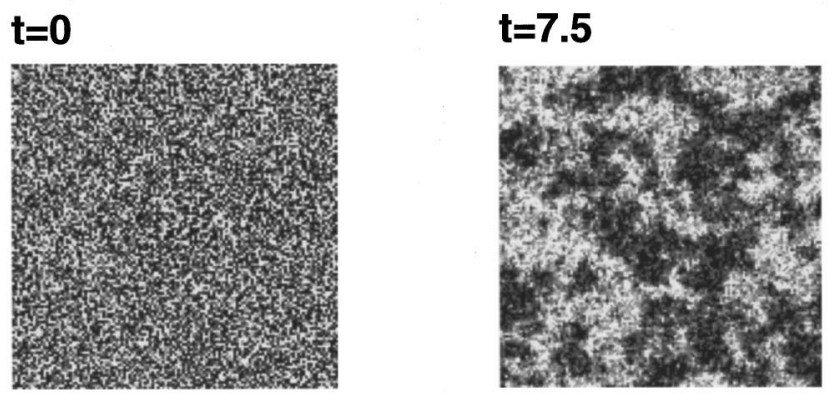

\section{$\mathbf{t}=\mathbf{0 . 2 5}$}

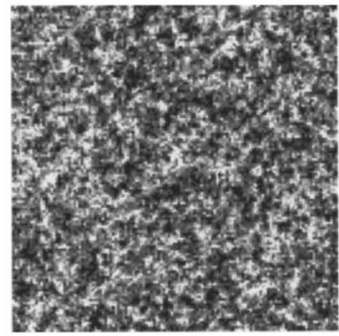

$t=1.0$

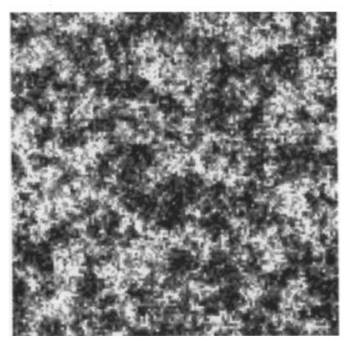

$t=15.0$

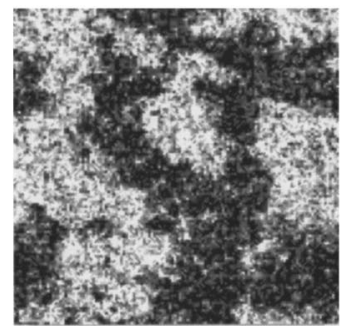

$t=60.0$

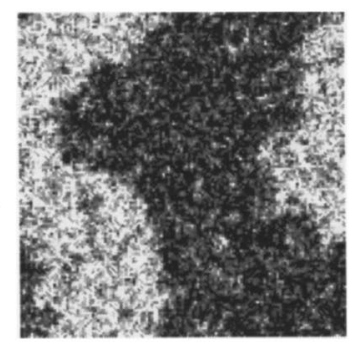

FIG. 2. Time evolution of domains starting in a completely random initial configuration toward an ordered phase for the spatially extended model given by Eqs. (8) and (29) on a square lattice, $\left(L=128, \sigma^{2}=4.00\right.$, and $\left.D=20\right)$. Dark areas correspond to positive values of the field $x_{\mathbf{r}}$, and light areas to negative values. Notice the initial development of small ordered regions which subsequently grow.

We can now understand a possible mechanism for the presence of a phase transition when coupling such scalar variables. Imagine that the short-time behavior can be described by a bistable potential $U_{\text {eff }}(x)$ according to the discussion in the previous paragraph and, hence, non-null symmetric states develop initially. Then, if the spatial coupling is sufficiently strong, it is possible that these non-null states couple to form local ordered regions which might subsequently coarsen and grow (see Fig. 2). This mechanism is the physical explanation of the existence of a phase transition in the spatially extended version of the system.

\section{MEAN-FIELD THEORY FOR SPATIALLY EXTENDED SYSTEMS}

We now consider spatially extended systems with multiplicative noise $[11,12,20-23]$. For simplicity, and in order to keep a clear connection with the zero-dimensional models discussed in Sec. II, we consider a lattice model with one spatially distributed scalar variable $x_{\mathbf{r}}$, with $\mathbf{r}$ determining the location of the lattice point under consideration. The time evolution of $x_{\mathbf{r}}$ is described by the following set of stochastic differential equations (we consider a hypercubic lattice in dimension $d$ with lattice spacing $a_{0}=1$ ):

$$
\dot{x}_{\mathbf{r}}=f\left(x_{\mathbf{r}}\right)+g\left(x_{\mathbf{r}}\right) \xi_{\mathbf{r}}+\frac{D}{2 d} \sum_{\mathbf{r}^{\prime} \in n(\mathbf{r})}\left(x_{\mathbf{r}^{\prime}}-x_{\mathbf{r}}\right),
$$

where $n(\mathbf{r})$ denotes the set of $2 d$ sites neighbor to $\mathbf{r}$, and $\left\{\xi_{\mathbf{r}}(t)\right\}$ are Gaussian noises, white in time and space, with zero mean and an autocorrelation function given by

$$
\left\langle\xi_{\mathbf{r}}(t) \xi_{\mathbf{r}^{\prime}}\left(t^{\prime}\right)\right\rangle=\sigma^{2} \delta_{\mathbf{r}, \mathbf{r}^{\prime}} \delta\left(t-t^{\prime}\right)
$$

( $\delta_{\mathbf{r}, \mathbf{r}^{\prime}}$ stands for a Kronecker $\delta$ function.) The last sum of Eq. (8) is, in the continuum limit, nothing but the usual diffusive Laplacian term $\nabla^{2} x_{\mathbf{r}}$. Equations of this kind are very general, and cover a multitude of different physical phenomena both in equilibrium and nonequilibrium problems. We focus in this paper on the steady-state properties of this system. However, the presence of multiplicative noise terms complicates matters significantly and, in fact, the multivariate steady-state probability $P^{\mathrm{st}}\left(\left\{x_{\mathbf{r}}\right\}\right)$ is only known in general for the case of additive noise, i.e., when $g(x)$ is a constant function.

The set of Eqs. (8) and (9) are equivalent to the following Fokker-Planck equation:

$$
\begin{aligned}
\partial_{t} P\left(\left\{x_{\mathbf{r}}\right\}, t\right) & =\sum_{\mathbf{r}}\left[\frac{\partial}{\partial x_{\mathbf{r}}}\left\{\left[-f\left(x_{\mathbf{r}}\right)+\frac{D}{2 d} \sum_{\mathbf{r}^{\prime} \in n(\mathbf{r})}\left(x_{\mathbf{r}}-x_{\mathbf{r}^{\prime}}\right)\right] P\left(\left\{x_{\mathbf{r}}\right\}, t\right)\right\}\right. \\
& \left.+\frac{\sigma^{2}}{2} \frac{\partial}{\partial x_{\mathbf{r}}}\left\{g\left(x_{\mathbf{r}}\right) \frac{\partial}{\partial x_{\mathbf{r}}}\left[g\left(x_{\mathbf{r}}\right) P\left(\left\{x_{\mathbf{r}}\right\}, t\right)\right]\right\}\right] .
\end{aligned}
$$

By integrating Eq. (10) over all variables with the exception of $x_{\mathbf{r}}$ (and assuming that the steady-state properties are uniform), one obtains the following exact steady-state equation for the one-site probability:

$$
\begin{aligned}
0= & \frac{\partial}{\partial x_{\mathbf{r}}}\left[-f\left(x_{\mathbf{r}}\right)+D\left[x_{\mathbf{r}}-E\left(x_{\mathbf{r}}\right)\right]\right. \\
& \left.+\frac{\sigma^{2}}{2} g\left(x_{\mathbf{r}}\right) \frac{\partial}{\partial x_{\mathbf{r}}} g\left(x_{\mathbf{r}}\right)\right] P^{\mathrm{st}}\left(x_{\mathbf{r}}\right),
\end{aligned}
$$

where

$$
E(y)=\left\langle x_{\mathbf{r}^{\prime}} \mid x_{\mathbf{r}}=y\right\rangle=\int d x_{\mathbf{r}^{\prime}} x_{\mathbf{r}^{\prime}} P^{\mathrm{st}}\left(x_{\mathbf{r}^{\prime}} \mid x_{\mathbf{r}}=y\right), \quad \mathbf{r}^{\prime} \in n(\mathbf{r})
$$

represents the steady-state conditional average of $x_{\mathbf{r}^{\prime}}$ at a neighboring site $\mathbf{r}^{\prime} \in n(\mathbf{r})$, given that $x_{\mathbf{r}}$ at site $\mathbf{r}$ takes the value $x_{\mathbf{r}}=y$. The solution to Eq. (11) is readily found (we drop the subscript $\mathbf{r}$ for simplicity of notation), 


$$
\begin{aligned}
& P^{\mathrm{st}}(x) \\
& =Z^{-1} \exp \left\{\int_{0}^{x} d y \frac{f(y)-\frac{\sigma^{2}}{2} g(y) g^{\prime}(y)-D[y-E(y)]}{\frac{\sigma^{2}}{2} g^{2}(y)}\right\},
\end{aligned}
$$

where $Z$ is a normalization constant. This result is exact, but we still have to determine the unknown function $E(y)$.

We start by considering in this section the simplest approximation [12], which is analogous to the traditional Weiss mean-field approach from the theory of equilibrium critical phenomena and which has also been applied successfully in several other stochastic problems [24-35]. In this approximation, one neglects the correlation between neighboring sites so that $E(y)=\langle x\rangle$, independent of $y$. Note that, the steady-state probability is now a function of $\langle x\rangle$, cf. Eq. (13). The value of $\langle x\rangle$ follows from a self-consistent relation arising from its very definition,

$$
\langle x\rangle=\int_{-\infty}^{+\infty} d x x P^{\mathrm{st}}(x) \equiv F(\langle x\rangle) .
$$

Since this is a complicated nonlinear equation in $\langle x\rangle$, the appearance of multiple solutions cannot be excluded, thus suggesting the possibility of breaking the ergodicity associated with the presence of a phase transition in the model.

Even though an exact analysis of Eq. (14) is difficult without specifying the explicit form of the functions $f$ and $g$, one can extract precious information by considering the strong-coupling limit $D \rightarrow \infty$. Using the saddle-point approximation, one finds that Eq. (14) reduces to the following simple equation for $\langle x\rangle$ :

$$
f(\langle x\rangle)+\frac{\sigma^{2}}{2} g(\langle x\rangle) g^{\prime}(\langle x\rangle)=0 .
$$

It is instructive to derive this result in a different way. One easily verifies that the evolution equation for the first moment $\left\langle x_{\mathbf{r}}(t)\right\rangle=\langle x(t)\rangle$ is identical in form to that for the zerodimensional system, i.e., it is given by Eq. (6). At the steadystate and considering the limit $D \rightarrow \infty$, one can neglect the fluctuations of the variable $x$ around its average value, and concludes that the steady-state equation for the first moment reduces to Eq. (15), which is thus exact in this limit.

Referring to the discussion in Sec. II, we conclude that in the limit $D \rightarrow \infty$ the system will undergo a second-order phase transition if the corresponding zero-dimensional model displays a linear instability in its short-time dynamics. The physical content of this conclusion is clear: when the system is strongly coupled, the short-time instability of the trajectory is the driving force behind the nonequilibrium phase transition. The criterion of short-time linear instability has been mentioned in other theoretical [22] and numerical [34] findings. However, we stress that it only holds as an approximation for finite values of $D$, and that it can completely break down for small values of $D$, cf. the example in Sec. V. As an interesting corollary, we return to the discussion of Sec. II to conclude that we expect pure noise-induced phase transitions in models for which the noise intensity has a minimum at the reference state and precisely not in models whose zerodimensional version displays a noise-induced transition (e.g., not in Hongler's model). This probably explains why the existence of noise-induced phase transitions was not discovered earlier (see also Ref. [36]).

\section{CORRELATION FUNCTION APPROACH}

We now present a more sophisticated approach, which involves the approximate calculation of the spatial correlation function. The method is an adaptation to multivariate Fokker-Planck equations of a technique developed in the context of multivariate Master equations [37,38]. The starting point is the following ansatz for the conditional average:

$$
\left\langle x_{\mathbf{r}^{\prime}} \mid x_{\mathbf{r}}=y\right\rangle=\int d x_{\mathbf{r}^{\prime}} x_{\mathbf{r}^{\prime}} P^{\mathrm{st}}\left(x_{\mathbf{r}^{\prime}} \mid x_{\mathbf{r}}=y\right)=\langle x\rangle+c_{\mathbf{r r}^{\prime}}(y-\langle x\rangle),
$$

where $c_{\mathbf{r r}^{\prime}}=\left\langle\delta x_{\mathbf{r}} \delta x_{\mathbf{r}^{\prime}}\right\rangle /\left\langle\delta x^{2}\right\rangle \quad(\delta x=x-\langle x\rangle)$ is the spatial correlation coefficient between sites $\mathbf{r}$ and $\mathbf{r}^{\prime}$. The system is assumed to be statistically homogeneous, so that single-site averages are independent of the specific location (and hence the subscript denoting the location will be dropped). In particular, the ansatz (16) implies that

$$
E(y)=\langle x\rangle+c(y-\langle x\rangle)
$$

where $c$ is the nearest-neighbor correlation coefficient $c=c_{\mathbf{r r}^{\prime}}$ with $\mathbf{r}^{\prime} \in n(\mathbf{r})$. Note that the mean-field approximation corresponds to the choice $c=0$. The variable $c$ appears as a second unknown in the explicit form of the steady-state probability, cf. Eqs. (13) and (17), and its value can be found self-consistently as follows. The ansatz (16) implies the following property for any function $\phi$ :

$$
\left\langle x_{\mathbf{r}^{\prime}} \phi\left(x_{\mathbf{r}}\right)\right\rangle=\langle x\rangle\langle\phi(x)\rangle+c_{\mathbf{r r}}\langle\delta x \phi(x)\rangle,
$$

which, in combination with the Fokker-Planck equation (10), leads to the following closed equation for the correlation function $c_{\mathbf{r r}}$ for a cubic lattice in dimension $d$ :

$$
\begin{array}{r}
\frac{D}{2 d}\left[\sum_{\mathbf{r}^{\prime \prime} \in n(\mathbf{r})}\left(c_{\mathbf{r} \mathbf{r}^{\prime}}-c_{\mathbf{r}^{\prime} \mathbf{r}^{\prime}}\right)+\sum_{\mathbf{r}^{\prime \prime} \in n\left(\mathbf{r}^{\prime}\right)}\left(c_{\mathbf{r r}^{\prime}}-c_{\mathbf{r} \mathbf{r}^{\prime \prime}}\right)\right] \\
=\beta \delta_{\mathbf{r}, \mathbf{r}^{\prime}}-2 \gamma c_{\mathbf{r r}^{\prime}},
\end{array}
$$

with $\beta$ and $\gamma$ given by

$$
\begin{gathered}
\beta=\frac{\sigma^{2}\left\langle g^{2}(x)\right\rangle}{\left\langle\delta x^{2}\right\rangle}, \\
\gamma=-\frac{\left\langle\delta x\left[f(x)+\frac{\sigma^{2}}{2} g(x) g^{\prime}(x)\right]\right\rangle}{\left\langle\delta x^{2}\right\rangle} .
\end{gathered}
$$

In deriving Eq. (18), we have used the fact that $\langle f(x)\rangle+\left(\sigma^{2} / 2\right)\left\langle g(x) g^{\prime}(x)\right\rangle=0$ at the steady-state, cf. Eq. (6). Obviously, $\beta>0$, and it can be also proved that $\gamma>0$ if $c<1$. 
Since the system is space-translational invariant at the steady-state, the correlation function can be written in terms of relative coordinates, i.e., $c_{\mathbf{r r}^{\prime}}=c\left(\mathbf{r}-\mathbf{r}^{\prime}\right)=c\left(\mathbf{r}^{\prime}-\mathbf{r}\right)$. Using this property, Eq. (18) can be rewritten as

$$
\frac{D}{d} \sum_{\mathbf{r}^{\prime} \in n(\mathbf{r})}\left[c\left(\mathbf{r}^{\prime}\right)-c(\mathbf{r})\right]=2 \gamma c(\mathbf{r})-\beta \delta_{\mathbf{r}, \mathbf{0}} .
$$

A closed expression for the correlation function $c(\mathbf{r})$ can be obtained in the case of a square lattice, $d=2$. By taking the Fourier transform of Eq. (20) one finds

$$
D\left[\cos \left(k_{x}\right)+\cos \left(k_{y}\right)-2\right] \widetilde{c}(\mathbf{k})=2 \gamma \widetilde{c}(\mathbf{k})-\beta,
$$

from which the Fourier transform $\widetilde{c}(\mathbf{k})$ of the correlation function is readily obtained. In order to perform the inverse Fourier transform, the following identity is helpful:

$$
\begin{aligned}
\int_{0}^{\infty} d z e^{-z t} I_{l}(z) I_{m}(z)= & \frac{1}{\pi^{2}} \int_{0}^{\pi} d k_{x} \\
& \times \int_{0}^{\pi} d k_{y} \frac{\cos \left(l k_{x}\right) \cos \left(m k_{y}\right)}{t-\cos \left(k_{x}\right)-\cos \left(k_{y}\right)},
\end{aligned}
$$

where $I_{l}(z)$ is the modified Bessel function. One concludes that

$$
c(\mathbf{r})=\frac{\beta}{D} \int_{0}^{\infty} d z e^{-2 z(1+\gamma / D)} I_{l}(z) I_{m}(z) \text { for } \mathbf{r}=l \mathbf{e}_{x}+m \mathbf{e}_{y},
$$

where $\left(\mathbf{e}_{x}, \mathbf{e}_{y}\right)$ is the unit cell of the square lattice.

A nearest neighbor in the square lattice has coordinates $l=1$ and $m=0$ (or, equivalently, $l=0$ and $m=1$ ), and one can perform the Laplace transform appearing in Eq. (22) explicitly (formula 12.9 in Ref. [39]) obtaining the following result for the quantity $c$ :

$$
c=-\frac{\beta}{2 D}+\frac{\beta}{\pi D} K\left[\left(\frac{D}{\gamma+D}\right)^{2}\right],
$$

where $K$ is the complete elliptic integral of the first kind. This expression can be further simplified by noting that $c=1-\beta / 2 D+\gamma / D$ which follows directly from Eq. (18) for the choice $\mathbf{r}=\mathbf{r}^{\prime}$. By elimination of $\beta$, one obtains the following final result for $c$ :

$$
c=\frac{\gamma+D}{D} \frac{K\left[\left(\frac{D}{\gamma+D}\right)^{2}\right]-\frac{\pi}{2}}{K\left[\left(\frac{D}{\gamma+D}\right)^{2}\right]} .
$$

The averages appearing in the definition of $\gamma$, cf. Eq. (19), have to be calculated with respect to the steady-state probability given by Eq. (13), which itself depends on $\langle x\rangle$ and $c$. As a result, Eqs. (14) and (24) form a set of two nonlinear self-consistent relations determining the values of $\langle x\rangle$ and $c$. When multiple solutions to these equations are found, one again expects that the system undergoes a phase transition.

We also mention the following result for the spatial correlation function along the axis of the square lattice:

$$
c_{l}=c\left(l \mathbf{e}_{x}\right)=\frac{\beta}{\pi D} \int_{0}^{\pi} d k_{x} \frac{\cos \left(l k_{x}\right)}{\sqrt{\left[2(1+\gamma / D)-\cos \left(k_{x}\right)\right]^{2}-1}} .
$$

Using the relation $1+2 \sum_{l=1}^{\infty} \cos \left(l k_{x}\right)=\pi \delta\left(k_{x}\right)$, one obtains the following simple result for the spatial correlation length $\lambda$ :

$$
\lambda \equiv \sum_{l=0}^{\infty} c_{l}=\frac{\beta}{4 D}\left[\frac{\gamma}{D}\left(1+\frac{\gamma}{D}\right)\right]^{-1 / 2}+\frac{1}{2} .
$$

At this point, a comment about the limitations of the present approach is in place. As it is clear from the previous result, the divergence of the correlation length requires that $\gamma \rightarrow 0$. On the other hand, it is also clear from Eq. (24) that in this limit $c=1$, and in fact $c(\mathbf{r})=1, \forall \mathbf{r}$. The reason of this behavior is that the decay of the spatial correlations is described by a single constant parameter $\gamma$, cf. Eq. (18). In a more sophisticated approximation, this would no longer be true and a less trivial appearance of long-range correlations would become possible. In the example that we will treat in Sec. V, we find that the parameter $\gamma$ never converges to 0 , even at a phase transition point. The present method is thus of no use in describing long-range spatial correlations. Its main virtue is to give improved results, when compared to the mean-field theory, for quantities such as the location of the critical point and the local probability density.

Finally, we note that the ansatz in Eq. (16) is exact if $\mathbf{r}$ and $\mathbf{r}^{\prime}$ are correlated Gaussian variables. This condition is verified for a linear Fokker-Planck equation $[f(x)=-x$ and $g(x)=1]$. The above-derived expressions are therefore exact for the linear model. One has in this case that $\langle x\rangle=0$, $\gamma=1$, and $\beta=2[1+(1-c) D]$, with the following final results for $c$ and $\lambda$ :

$$
\begin{gathered}
c=\frac{1+D}{D} \frac{K\left[\left(\frac{D}{1+D}\right)^{2}\right]-\frac{\pi}{2}}{K\left[\left(\frac{D}{1+D}\right)^{2}\right]}, \\
\lambda=\frac{1}{2}+\frac{1+(1-c) D}{D \sqrt{(1+2 / D)^{2}-1}} .
\end{gathered}
$$

\section{PURE NOISE-INDUCED PHASE TRANSITION}

The results obtained in Sec. IV are general; they can be applied for any choice of the functions $f(x)$ and $g(x)$. In this paper, however, we want to discuss in detail the case of pure noise-induced phase transitions. We will focus here on the prototype model that was introduced in Ref. [11], namely, the set of Eqs. (8) with the choice

$$
f(x)=-x\left(1+x^{2}\right)^{2}, \quad g(x)=1+x^{2} .
$$

We are inclined to believe that it is the simplest possible model that exhibits such a transition, and possibly corresponds to a kind of "normal form unfolding."

As a first approach to understanding this model, in Fig. 3, we show the phase diagrams as predicted by the mean-field theory and the correlation function approach (CFA) described in Secs. III and IV, respectively. These have been 


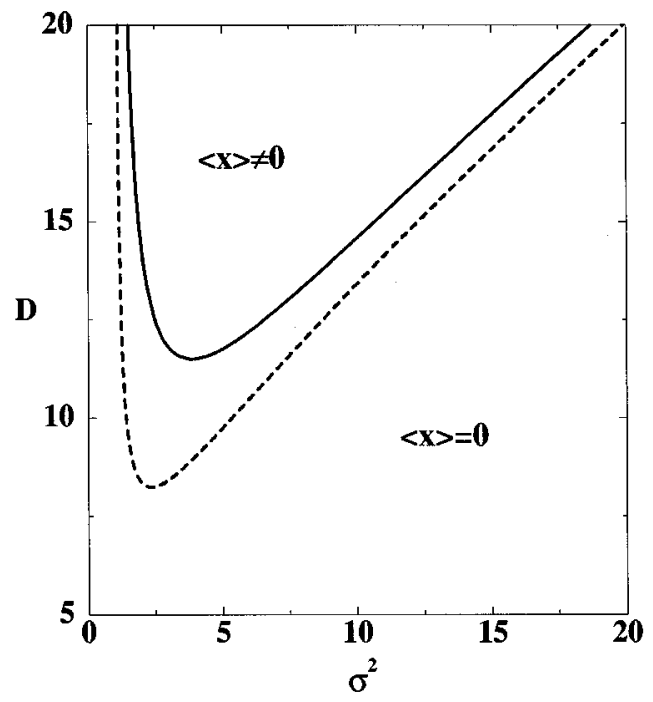

FIG. 3. Phase diagram for the noise-induced phase transition as predicted by the mean-field theory (dashed line), cf. Eq. (14), and the CFA (full line), based on Eqs. (14) and (24) for dimension $d=2$.

obtained by numerical solution of the self-consistency relations Eqs. (14) and (24). The most important feature shown in Fig. 3 is the prediction of the existence of a symmetrybreaking phase corresponding to a solution of the selfconsistent equations with $\langle x\rangle \neq 0$. Both the mean-field theory and the CFA predict the appearance of such a phase, although they vary slightly in the region of parameters for which one expects the ordered phase to exist. According to these approximate theories, the ordered phase appears through a second-order phase transition for a sufficiently strong spatial coupling $D$, and at a finite critical value of the noise intensity $\sigma_{c}^{2}$. There is no phase transition if the spatial coupling $D$ is less than some critical value. This agrees well with our intuitive explanation of the transition given in Sec. II: if the coupling is not strong enough, and local ordering cannot be induced in the early evolution, the late-time distribution will be governed instead by the maxima $\bar{x}$ of the steady-state probability density, Eq. (5), which in this case is $\bar{x}=0$. If we turn now to the limit of very strong spatial coupling, when $D \rightarrow \infty$, the location of the critical point at $\sigma^{2}=1$ is in agreement with the linear instability criterion mentioned in Sec. II. Another interesting, although certainly not surprising, feature of the transition, is that as one increases further the noise intensity, the ordered phase disappears through another second-order phase transition. This second, reentrant, transition shows that the more "traditional" effect of the noise, namely, the ability to destroy ordered states, is also present in our model.

If we take, for instance, $D=20$, the order parameter $\langle x\rangle$ takes nonzero values only for noise intensity values in the interval $\left(\sigma_{c_{1}}^{2}, \sigma_{c_{2}}^{2}\right)$. The mean-field theory predicts the phase transition points at $\sigma_{c_{1}}^{2} \approx 1.11$ and $\sigma_{c_{2}}^{2} \approx 19.1$, while the CFA yields a somewhat narrower region for the ordered phase, namely $1.50<\sigma^{2}<18.7$. It is expected that the mean-field approach and, to a lesser extent, the CFA, overestimate the size of the ordered region.

In order to check the validity of these mean-field type

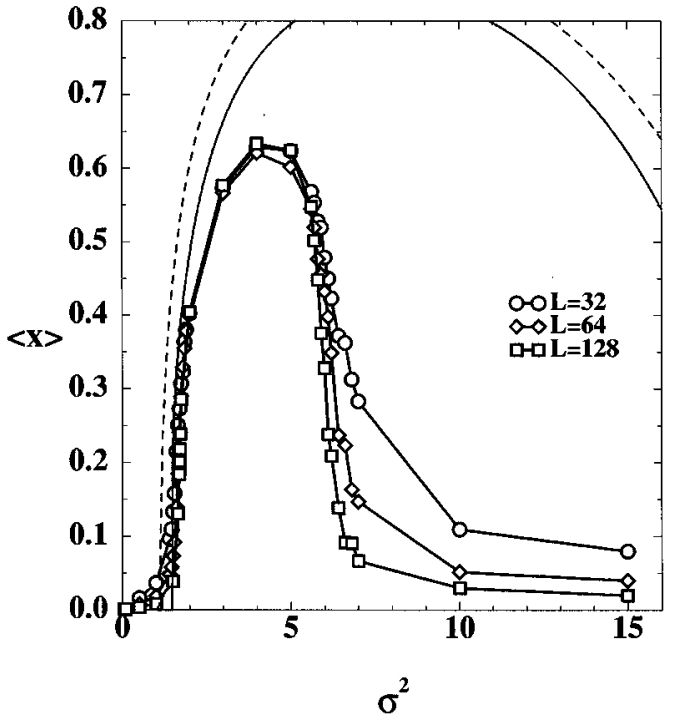

FIG. 4. Order parameter $\langle x\rangle$ vs intensity of the noise for $D=20$, according to the mean-field theory (dashed line), the CFA for $d=2$ (full line), and $2 d$ simulations for system sizes $32 \times 32$ (circles), $64 \times 64$ (diamonds), and $128 \times 128$ (squares). Notice that although the general features of mean-field approximations agree with the simulation result, they tend to overestimate the ordered region.

approximations and also to gain an understanding of this phenomenon of a noise-induced phase transition, we have performed numerical simulations of the model defined by Eqs. (8) and (29) on a square lattice (see the Appendix for details of the simulations). The simulations confirm qualitatively all the predictions of the mean-field approaches, and give us more accurate data about the transition points. In Fig. 4 we plot the order parameter $\langle x\rangle$ as a function of $\sigma^{2}$ for $D=20$ according to the two mean-field-type theories developed earlier together with the simulation results for various system sizes. The simulation data indeed confirm the existence of both phase transitions, but the ordered phase appears in a smaller interval $1.71<\sigma^{2}<5.8$. The latter values have been obtained on the basis of finite-size scaling analysis, cf. Sec. VI.

\section{CRITICAL PHENOMENA}

The pure noise-induced phase transition discussed in Sec. $\mathrm{V}$ appears to be an interesting phenomenon. The question therefore arises as to whether this transition shares the usual features of equilibrium phase transitions and, additionally, whether it belongs to any of the existing universality classes. In this sense, a theoretical argument has recently been put forward indicating that the critical properties are those of the Ising universality class [40]. To investigate these points, we have performed extensive computer simulations in the vicinity of both the entrant and reentrant critical points for $D=20$ for two-dimensional systems of size $L \times L$ for values of $L$ ranging in size between $L=10$ and 128. Apart from the order parameter $m=\left|L^{-2} \Sigma_{\mathbf{r}} x_{\mathbf{r}}\right|$, and the correlation coefficient, $c$, we also measure higher-order moments, as well as time and spatial correlations. The results are collected in Figs. 4-11. One clearly recognizes all the trademarks of 


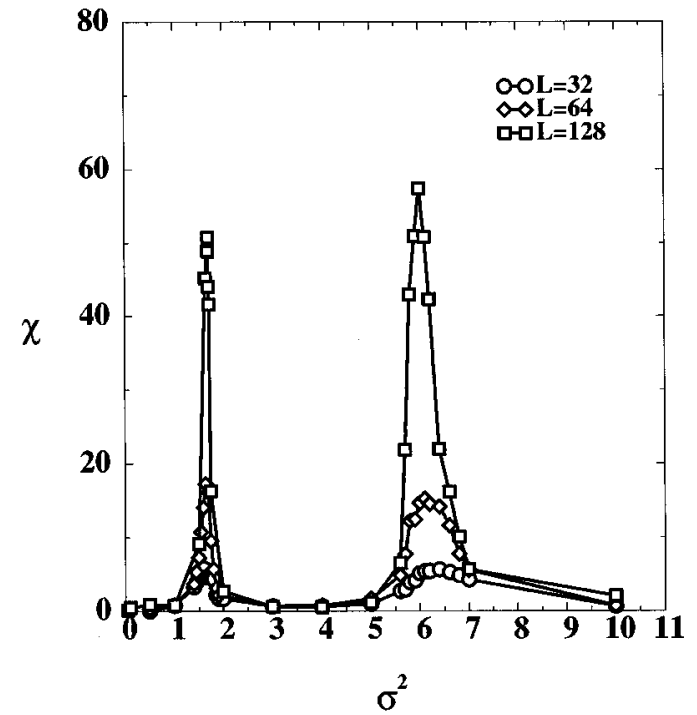

FIG. 5. Susceptibility, $\chi=\left(L^{2} / \sigma^{2}\right)\left[\left\langle m^{2}\right\rangle-\langle m\rangle^{2}\right]$, as a function of $\sigma^{2}$ for system sizes $32 \times 32$ (circles), $64 \times 64$ (diamonds), and $128 \times 128$ (squares). The peaks clearly show the enhancement of fluctuations around the two critical points.

second-order phase transitions: divergence of fluctuations (Fig. 5), scaling properties (Figs. 7 and 8), long-range spatial correlations (Fig. 9), and critical slowing down (Fig. 10). We now discuss each of these topics in some detail.

In an equilibrium second-order phase transition, the relative fluctuations of the order parameter (susceptibility) and

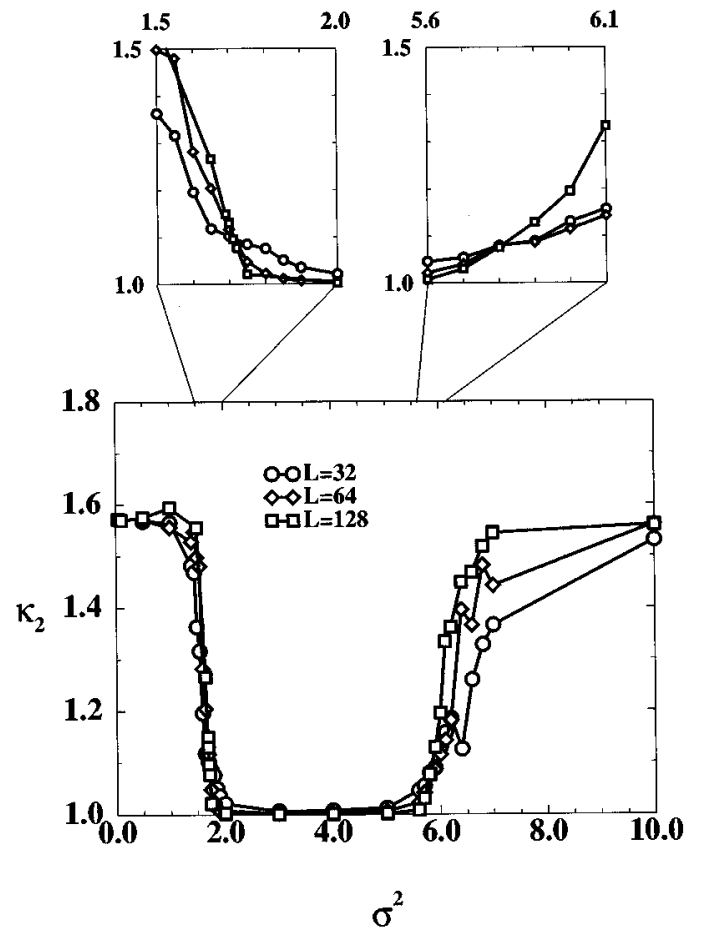

FIG. 6. Second-order cumulant $\kappa_{2}=\left\langle m^{2}\right\rangle /\langle m\rangle^{2}$ as a function of $\sigma^{2}$ for system sizes $32 \times 32$ (circles), $64 \times 64$ (diamonds), and $128 \times 128$ (squares). The curves cross at $\sigma^{2} \approx 1.71$ and $\sigma^{2} \approx 5.8$, which, according to the finite-size scaling theory, are identified as the location of the critical points (see the text).
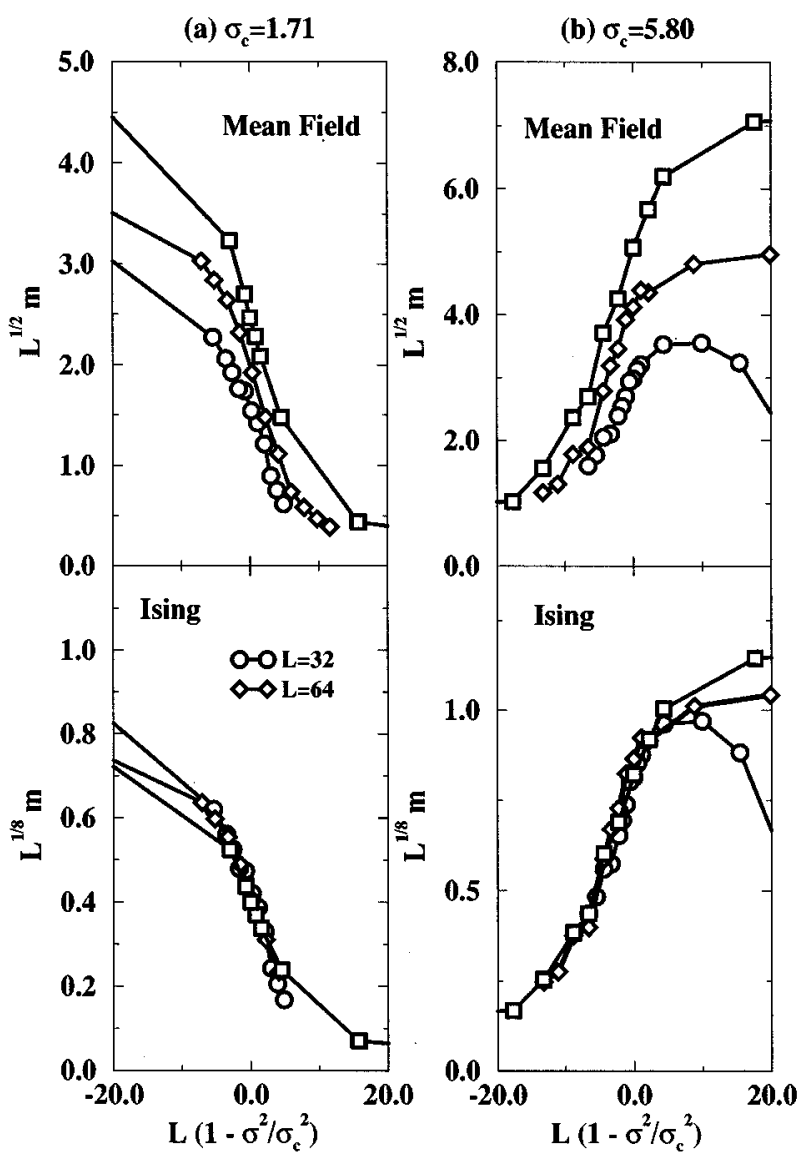

FIG. 7. Plot of $\langle m\rangle L^{v}$ vs $\left(1-\sigma^{2} / \sigma_{c}^{2}\right) L^{u}$ in order to check the prediction of finite-size scaling of the order parameter $\langle m\rangle$ for the entrant [plots (a)] and the reentrant [plots (b)] transitions. We use in this figure the Ising $\left(u=1, v=\frac{1}{8}\right)$ and mean-field $\left(u=1, v=\frac{1}{2}\right)$ critical exponents. The quality of the scaling is certainly superior for the Ising exponents than for the mean-field ones.

energy (specific heat) diverge with characteristic critical exponents. In our model, we define a "susceptibility" $\chi$ as a suitable measure of the fluctuations of the order parameter

$$
\chi \equiv \frac{L^{2}}{\sigma^{2}}\left[\left\langle m^{2}\right\rangle-\langle m\rangle^{2}\right]
$$

this definition, and more specifically, the presence of the $\sigma^{2}$ term in the denominator is the equivalent of the usual definition $\chi=L^{2}\left[\left\langle m^{2}\right\rangle-\langle m\rangle^{2}\right] / k_{B} T$ for thermal systems. In Fig. 5 we plot $\chi$ as a function of noise intensity $\sigma^{2}$ for different system sizes. The enhancement of fluctuations near the two critical points is clear. In the case of equilibrium phase transitions, the susceptibility at the critical point $\chi_{c}$ only diverges in the thermodynamic limit $L \rightarrow \infty$. For finite systems, the theory of finite-size scaling [41] tells us that the critical value $\chi_{c}$ increases as a suitable power of the system size, namely, $\chi_{c} \sim L^{y}$, with the value of $y$ related to the values of the critical exponents. Since in Fig. 5 it is obvious that fluctuations also grow with system size, it is very tempting to try to analyze our data using the standard techniques that have been so successful for equilibrium phase transitions. We now briefly review the main predictions of finite-size scaling theory that are relevant to our study. For a thermal 


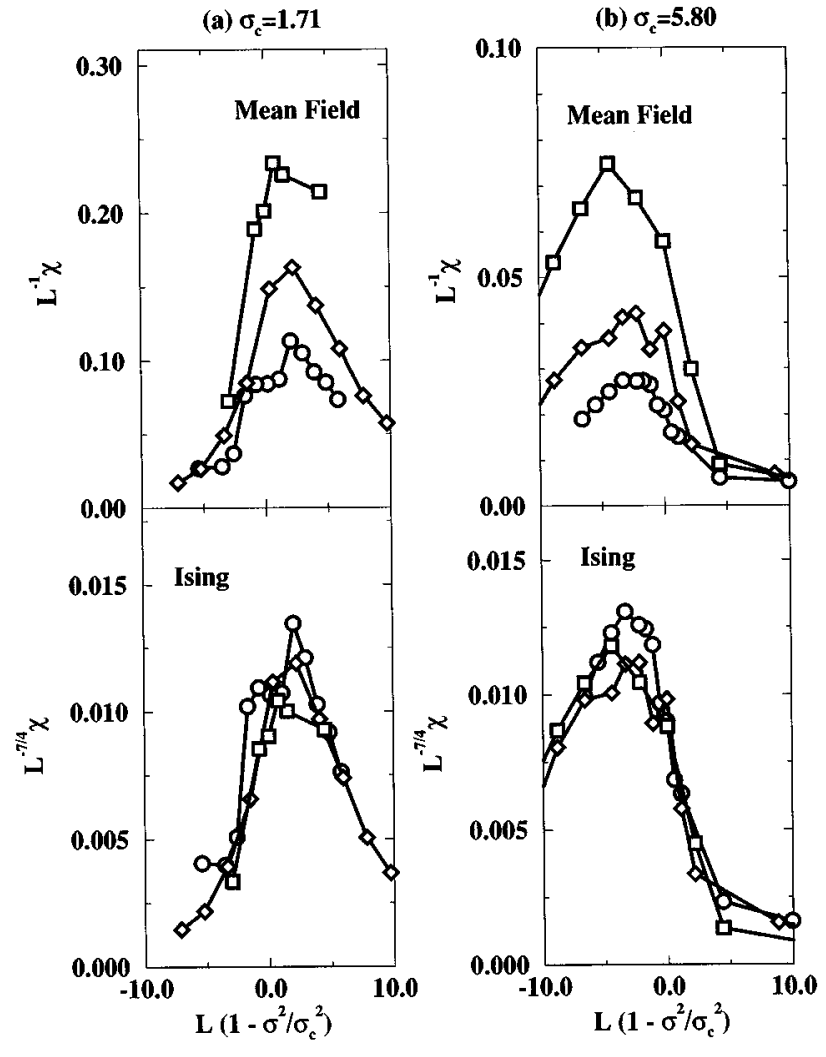

FIG. 8. Plot of $\chi L^{2 v-2}$ vs $\left(1-\sigma^{2} / \sigma_{c}^{2}\right) L^{u}$ in order to check the prediction of finite-size scaling of the "susceptibility" $\chi$ for the entrant [plots (a)] and the reentrant [plots (b)] transitions. We use in this figure the Ising $\left(u=1, v=\frac{1}{8}\right)$ and mean-field $\left(u=1, v=\frac{1}{2}\right)$ critical exponents. As in the case of the order parameter, Fig. 7, a better scaling is obtained when using the Ising exponents.

phase transition the order parameter $m$ is a function of temperature $T$ and system size $L$. Finite-size scaling theory predicts that near a critical point the average of the $k$ th-order moment of $m$ is a homogeneous function of its arguments, namely $[42,43]$,

$$
\left\langle m^{k}\right\rangle=L^{-k v} \widetilde{m}_{k}\left(\epsilon L^{u}\right),
$$

where $\epsilon \equiv 1-T / T_{c}$ is a measure of the distance to the critical point, $\widetilde{m}_{k}$ is a scaling function, and $u$ and $v$ are critical exponents which take different values according to whether we are below the critical dimension where hyperscaling relations hold $(u=1 / \nu$ and $v=\beta / \nu)$ or above the critical dimension where mean-field exponents hold $(u=d / 2, v=d / 4)$. In the following, we will assume that finite-size scaling also holds for our system such that equivalent relations are valid with $\epsilon \equiv 1-\sigma^{2} / \sigma_{c}^{2}$ measuring the distance to the transition point. We will use the above expressions in order to locate the critical points $\sigma_{c}^{2}$ and also to compute the critical exponents $u$ and $v$, which in turn, will allow us to obtain the exponents $\nu$ and $\beta$.

A precise determination of the critical values of the noise intensity is obtained by focusing on the behavior of the second-order cumulant $\kappa_{2} \equiv\left\langle m^{2}\right\rangle /\langle m\rangle^{2}$. According to the previous finite-size scaling relation one finds

$$
\kappa_{2}\left(\sigma^{2}, L\right)=\widetilde{\kappa}_{2}\left(\epsilon L^{-v}\right) .
$$

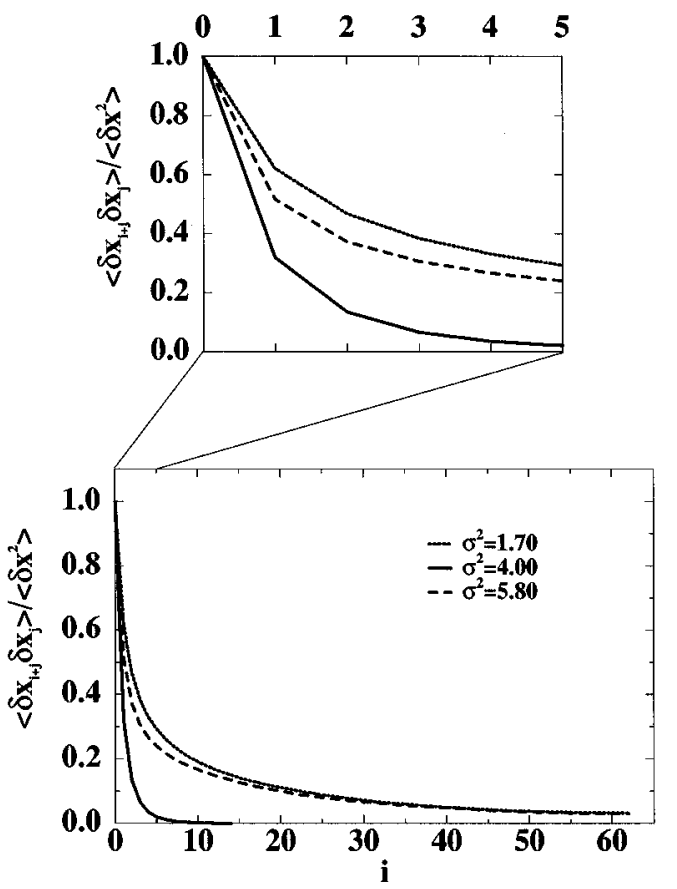

FIG. 9. Plot of the normalized spatial correlation function $c(i)=\left\langle\delta x_{i+j} \delta x_{j}\right\rangle /\left\langle\delta x^{2}\right\rangle$ in the vicinity of the critical points $\sigma_{c_{1}}^{2}=1.71, \sigma_{c_{2}}^{2}=5.8$ and in an intermediate value of $\sigma^{2}$ corresponding to the ordered phase region (we have used in this figure $D=20$, system size $L=128$ ). Notice the slow decay of the spatial correlation function near the two critical points.

For $\sigma^{2}=\sigma_{c}^{2}$, one has $\epsilon=0$ and the prediction is that $\kappa_{2}\left(\sigma_{c}^{2}, L\right)=\widetilde{\kappa}_{2}(0)$, i.e., a constant independent of the system size $L$. As a consequence, by plotting the second-order cumulants for different system sizes, we can determine the critical points as the ones in which the curves for different values of $L$ cross each other. By analyzing the cumulant data in this way (Fig. 6), we are able to locate the entrant critical point quite precisely at $\sigma^{2}=1.71 \pm 0.01$, while the reentrant transition is at $\sigma^{2}=5.8 \pm 0.1$.

For the order parameter scaling, the prediction is $\langle m\rangle=L^{-v} \widetilde{m}\left(\epsilon L^{u}\right)$, such that a plot of $\langle m\rangle L^{v}$ versus $\epsilon L^{u}$ should yield a curve independent of the system size. This is checked in Fig. 7. Although it is true that the statistical errors of the data do not allow a very precise determination of the critical exponents $u$ and $v$, it is shown in the figure that, both for entrant and reentrant transitions, scaling holds better if we use the $2 d$ Ising critical exponents $\beta=\frac{1}{8}$ and $\nu=1$ $\left(u=1\right.$ and $\left.v=\frac{1}{8}\right)$ than the mean-field ones $u=d / 2=1$ and $v=d / 4=\frac{1}{2}$. The same conclusions are reached when analyzing the finite-size scaling behavior of the susceptibility, which, according to its definition Eq. (30), should behave as $\chi=L^{d-2 v} \widetilde{\chi}\left(\epsilon L^{u}\right)$. In Fig. 8 we show that properly scaled susceptibility curves fall on top of each other rather well, when using the Ising critical exponents $d-2 v=\frac{7}{4}$ and $u=1$, whereas the quality of scaling is worse when using mean-field exponents $d-2 v=1$ and $u=1$. Note that, in a previous paper [11], we found a good fit at the entrant transition using mean-field exponents for smaller system sizes (up to $48 \times 48$ ). This discrepancy may be due to the fact that the regime of nonclassical behavior is located in a narrow 


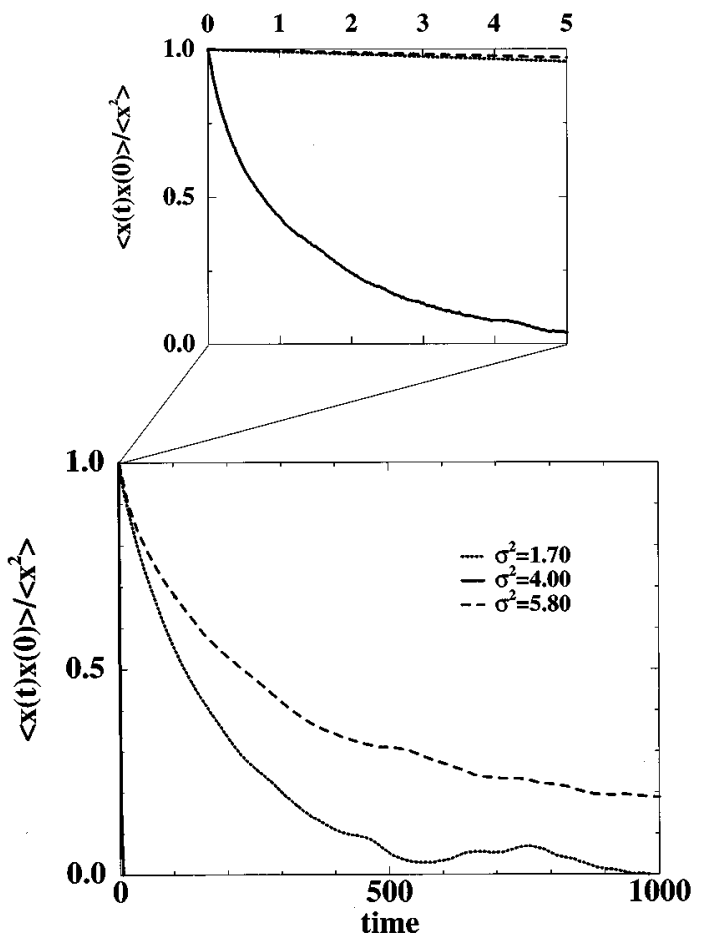

FIG. 10. Similar to Fig. 9 but for the temporal correlation function in the vicinity of the critical points and in the ordered phase region $(D=20$, system size $L=128)$.

neighborhood of the critical point that could not be investigated with these system sizes. Further studies will be necessary to determine unambiguously if both transitions (entrant and reentrant) belong to the same universality class of the Ising model.

Finally, the spatial and temporal correlation functions are represented in Figs. 9 and 10 for several values of $\sigma^{2}$. One clearly observes the appearance of long-range correlations in the vicinity of the two critical points, another signature of a phase transition for equilibrium systems. In Fig. 11, we plot the nearest-neighbor correlation coefficient $c$ as a function of $\sigma^{2}$, and compare it with the results obtained through the CFA, cf. Eqs. (24) and (27). As expected, the agreement is only good for small values of the noise intensity.

\section{PERSPECTIVES}

We have confirmed the existence of a pure noise-induced phase transition in the model introduced in Ref. [11], explained the role of the short-time instability of the single-site stochastic dynamics in generating the transition, and given evidence that its critical properties are compatible with those of Ising universality class (in $d=2$ ). These results open a number of perspectives for future research. First, the model that we introduced has been chosen for its mathematical simplicity, but it does not have a direct physical meaning. The intuitive arguments given in Sec. II, however, suggest that the pure noise-induced transitions will arise generically in systems with a multiplicative noise term, whose amplitude has a minimum in the reference state. It remains to be seen whether our model corresponds to a kind of "normal form unfolding', of such phase transitions. Second, we expect

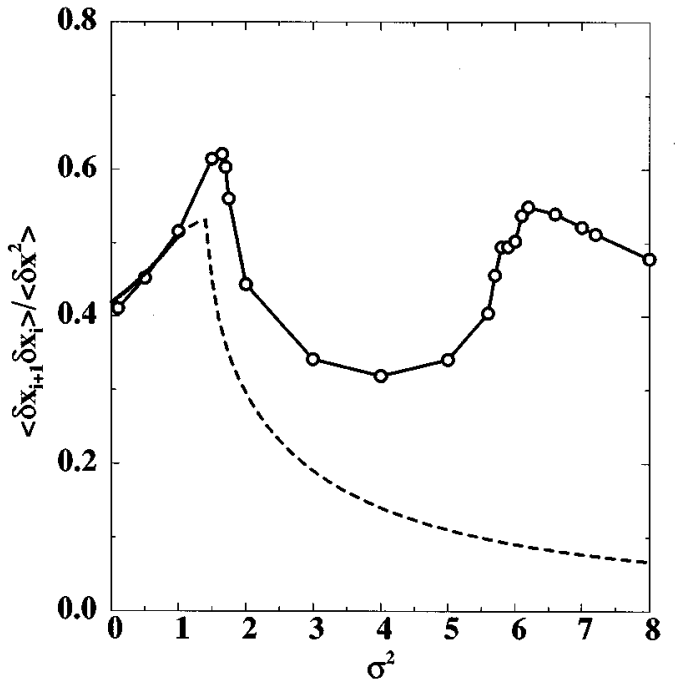

FIG. 11. Nearest-neighbor correlation coefficient $c$ [see Eq. (16)] vs $\sigma^{2}$ from simulations $(L=128)$ and theory, cf. Eq. (24). As expected, the agreement is only good for small values of the noise intensity $\sigma^{2}$.

that, even though our model seems to belong to the Ising universality class as far as its critical properties are concerned, the specific noise-induced mechanism by which order appears will reflect itself in the time-dependent properties such as nucleation phenomena or the response to external fields or symmetry-breaking boundary conditions. Third, it is clear that phase transitions of another order (first or higher order) can be generated [44]. In the case of a first-order transition, this would imply that the macroscopic state of the system would change dramatically when the intensity of the noise is varied across a threshold value. Fourth, more complicated pure noise-induced phase transitions, that break temporal symmetry [45], spatial symmetry [46] or both, can be constructed. Finally, we propose to make a search for or reevaluation of experiments in physical systems for which noise-induced shifts $[11,20]$, and pure noise-induced phase transitions may be relevant. Some cases have been documented in the literature of noise-induced shifts in the phase transition or bifurcation point, for example in photosensitive chemical reactions, subject to a fluctuating light intensity $[47,48]$, in liquid crystals $[49,50]$, and in the Raleigh-Benard instability with a fluctuating temperature at the plates [51]. Also, stochastic equations for spatially distributed systems with multiplicative noise have appeared recently in several contexts, including lasers [52], directed percolation [53], and other models for growth [54].

\section{ACKNOWLEDGMENTS}

This work was supported by NATO Grant No. CRG 950055. C.V.d.B. also acknowledges support from the Program on Inter-University Attraction Poles, Prime Minister's Office, Belgian Government and NFWO Belgium; J.M.R.P. from Dirección General de Investigación Científica y Técnica (DGICYT) (Spain), Project No. PB94-0388; R.T. from DGICYT Projects No. PB94-1167 and No. PB94-1172; and R.K. from Cray Research Inc. and Alabama Education and Research Network. Most of the computer simulations 
were carried out using Cray C90 at Alabama Supercomputer Center and the Connection Machine model 5E at Naval Research Laboratory.

\section{APPENDIX: COMPUTER SIMULATIONS}

A Monte Carlo simulation of the stochastic process (8) was performed for two-dimensional square lattices of various sizes up to $L=128$ with periodic boundary conditions. The stochastic differential equation for the variable at the $i$ th site $x_{i}$ is given by

$$
\frac{d x_{i}}{d t}=F_{i}(\mathbf{x})+G_{i}(\mathbf{x}) \xi_{i}(t), \quad i=1, \ldots, N=L^{2}
$$

where $\mathbf{x}=\left(x_{1}, \ldots, x_{N}\right)$, and

$$
\begin{gathered}
F_{i}(\mathbf{x})=f\left(x_{i}\right)-\frac{D}{4} \sum_{j \in n(i)}\left(x_{i}-x_{j}\right), \\
G_{i}(\mathbf{x})=g\left(x_{i}\right) .
\end{gathered}
$$

These equations were integrated using two different algorithms, the Milshtein and the Heun methods [55,56].

The Milshtein method allows us to advance forward in time by means of the recursion relations

$$
\begin{aligned}
x_{i}(t+\delta t)= & {\left[F_{i}(\mathbf{x}(t))+\frac{\sigma^{2}}{2} G_{i}(\mathbf{x}(t)) \frac{d G_{i}(\mathbf{x}(t)}{d x_{i}}\right] \delta t } \\
& +G_{i}(\mathbf{x}(t)) \sqrt{\sigma^{2} \delta t} \eta_{i}(t),
\end{aligned}
$$

where $\eta_{i}(t)$ are independent Gaussian random variables of zero mean and variance equal to 1 , and the second term is included because Eq. (A1) is interpreted in the Stratonovich sense. The order of numerical error in the Milshtein method is $\delta t$. Therefore, a small $\delta t$ (e.g., $\delta t=1 \times 10^{-4}$ for $\sigma^{2}=1$ ) must be used, while its computational effort per time step is relatively small. For large $\sigma$, where fluctuations are rapid and large, a longer integration period and a smaller $\delta t$ is necessary. The Milshtein method quickly becomes impractical.

The Heun method is based on the second-order RungeKutta method, and integrates the stochastic equation by a recursive equation

$$
\begin{aligned}
x_{i}(t+\delta t)= & x_{i}(t)+\frac{\delta t}{2}\left[F_{i}(\mathbf{x}(t))+F_{i}(\mathbf{y}(t))\right]+\frac{\sqrt{\sigma^{2} \delta t}}{2} \eta_{i}(t) \\
& \times\left[G_{i}(\mathbf{x}(t))+G_{i}(\mathbf{y}(t))\right],
\end{aligned}
$$

where

$$
y_{i}(t)=x_{i}(t)+F\left(x_{i}(t)\right) \delta t+G\left(x_{i}(t)\right) \eta_{i}(t) \sqrt{\sigma^{2} \delta t} .
$$

This method allows larger $\delta t$ than the Milshtein method, without a significant increase in computational effort per step. We used this method for $\sigma^{2}>2$.

The time step $\delta t$ has been chosen by a stability condition, and also such that averaged magnitudes do not depend on $\delta t$ within statistical errors. For $D=20$, for example, the necessary values for $\delta t$ vary between $\delta t=5 \times 10^{-4}$ for $\sigma^{2}=1$ and $\delta t=1 \times 10^{-5}$ for $\sigma^{2}=15$. The Gaussian random numbers necessary for the simulations were generated either by using the Box-Muller-Wiener algorithm or a very fast numerical inversion method [57]. The time evolution of the average value is carefully monitored until the stationary state is reached.

The order parameter is computed by

$$
\langle m\rangle=\left\langle\left\langle\left|\frac{1}{L^{2}} \sum_{i=1}^{N} x_{i}\right|\right\rangle_{\text {time }}\right\rangle_{\text {ensemble }},
$$

where \langle\rangle$_{\text {time }}$ and \langle\rangle$_{\text {ensemble }}$ indicate time average and ensemble average, respectively. The averaging time $T$ was chosen to be sufficiently longer than the correlation time, for example, $T \approx 2 \times 10^{4}\left(10^{8}\right.$ steps $)$ near the critical points. The ensemble average was taken over at least ten independent systems. Similarly, the susceptibility is evaluated as

$$
\left.\chi=\frac{L^{2}}{\sigma^{2}}\left\langle\left\langle\left(\frac{1}{L^{2}} \sum_{i=1}^{N} x_{i}\right)^{2}-\langle m\rangle^{2}\right\rangle\right\rangle_{\text {time }}\right\rangle \text { ensemble }
$$

Simulation of large systems $(128 \times 128)$ was too long for Cray C90 despite the code is mostly vectorized. Therefore, we used a massively parallel computer, the connection machine model $5 \mathrm{E}$ with 256 processors which appeared to be about ten times faster than Cray C90 for this particular application with the same programs.
[1] W. Horsthemke and R. Lefever, Noise-Induced Transitions (Springer-Verlag, Berlin, 1984).

[2] A. Schenzle and H. Brandt, Phys. Rev. A 20, 1628 (1979).

[3] H. Risken, The Fokker-Planck Equation (Springer-Verlag, Berlin, 1984).

[4] R. Graham and A. Schenzle, Phys. Rev. A 25, 1731 (1982).

[5] Noise in Nonlinear Dynamical Systems, edited by F. Moss and P. V. E. McClintock (Cambridge University Press, Cambridge, 1989).

[6] A. S. Mikhailov, Phys. Rep. 184, 307 (1989).

[7] L. Schimansky-Geier and Ch. Zulicke, Z. Phys. B 82, 157 (1991).

[8] M. O. Magnasco, Phys. Rev. Lett. 71, 1477 (1993); see also L. P. Faucheux, L. S. Bourdieu, P. D. Kaplan, and A. J. Libch- aber, Phys. Rev. Lett. 74, 1504 (1995) for more recent references.

[9] Instabilities and Chaos in Quantum Optics II, edited by N. B. Abraham, F. T. Arecchi, and L. A. Lugiato (Plenum, New York, 1988).

[10] Ch. Doering and C. Gadoua, Phys. Rev. Lett. 69, 2138 (1992).

[11] C. Van den Broeck, J. M. R. Parrondo, and R. Toral, Phys. Rev. Lett. 73, 3395 (1994).

[12] C. Van den Broeck, J. M. R. Parrondo, J. Armero, and A. Hernández-Machado, Phys. Rev. E 49, 2639 (1994).

[13] There exists an infinity of interpretations of stochastic differential equations with multiplicative noise, with, on one extreme, the mathematically minded Ito rule [corresponding to a diffusion term of the form $\left.\partial_{x}^{2}\left[g^{2}(x) P(x, t)\right]\right]$, and on the other 
extreme the kinetic definition [corresponding to a diffusion term of the form $\left.\partial_{x}\left[g^{2}(x) \partial_{x} P(x, t)\right]\right]$. Which interpretation is valid depends on the origin of the white noise $[14,15]$ and its interaction with eventual other fast degrees of freedom of the system [16].

[14] N. G. Van Kampen, Stochastic Processes in Physics and Chemistry (North-Holland, Amsterdam, 1992).

[15] R. E Mortensen, J. Stat. Phys. 1, 271 (1969).

[16] R. Graham and A. Schenzle, Phys. Rev. A 26, 1676 (1982).

[17] M. O. Hongler, Helv. Phys. Acta. 52, 280 (1979).

[18] D. Amit, Field Theory, the Renormalization Group and Critical Phenomena (World Scientific, Singapore, 1984).

[19] R. Toral and A. Chakrabarti, Phys. Rev. B 42, 2445 (1990).

[20] J. García-Ojalvo, A. Hernández-Machado, and J. M. Sancho, Phys. Rev. Lett. 71, 1542 (1993).

[21] L. Ramírez-Piscina, J. M. Sancho, and A. HernándezMachado, Phys. Rev. B 48, 119 (1993); 48, 125 (1993).

[22] A. Becker and L. Kramers, Phys. Rev. Lett. 73, 955 (1994).

[23] G. Grinstein, M. A. Muñoz, and T. Yuhai, Phys. Rev. Lett. 76, 4376 (1996).

[24] Y. Onodera, Prog. Theor. Phys. 44, 1477 (1970).

[25] M. Malek Mansour and G. Nicolis, J. Stat. Phys. 13, 197 (1975)

[26] R. Desai and R. Zwanzig, J. Stat. Phys. 19, 1 (1978).

[27] A. D. Bruce, Adv. Phys. 29, 111 (1980).

[28] R. A. Dawson, J. Stat. Phys. 31, 29 (1983).

[29] M. O. Hongler and R. Desai, J. Stat. Phys. 32, 585 (1983).

[30] G. Dewel, P. Borckmans, and D. Walgraef, Phys. Rev. A 31, 1983 (1985).

[31] O. T. Valls and G. F. Mazenko, Phys. Rev. B 34, 7941 (1986).

[32] M. Shiino, Phys. Rev. A 36, 2393 (1987).

[33] K. Kaneko, Phys. Rev. Lett. 65, 1391 (1990).

[34] J. García-Ojalvo, J. M. Sancho, and L. Ramírez-Piscina, Phys. Lett. A 168, 35 (1992).

[35] P. Jung, U. Behn, E. Pantazelou, and F. Moss, Phys. Rev. A 46, R1709 (1992).

[36] Ch. Doering, Phys. Lett. A 122, 133 (1987).

[37] M. Malek Mansour and J. Houard, Phys. Lett. 70A, 366 (1979).

[38] C. Van den Broeck and M. Malek Mansour, in Instabilities and Nonequilibrium Structures, edited by E. Tirapegui and W. Zeller (Kluwer, Dordrecht, 1993), p. 49.

[39] G. E. Roberts and H. Kaufman, Table of Laplace Transforms (Saunders, Philadelphia, 1966).

[40] S. Ramaswamy, R. Pandit, and R. Lahiri, Phys. Rev. Lett. 75, 4786 (1995).

[41] Finite-Size Scaling, edited by J. L. Cardy (North-Holland, Amsterdam, 1988)

[42] K. Binder and D. W. Heermann, Monte Carlo Simulation in Statistical Physics, (Springer-Verlag, Berlin, 1988).

[43] H.-P. Deutsch, J. Stat. Phys. 67, 1039 (1992).

[44] R. Muller, K. Lippert, A. Kuhnel and V. Behn (unpublished).

[45] S. H. Park and S. Kim, Phys. Rev. E 53, 3425 (1996).

[46] J. M. R. Parrondo, C. Van den Broeck, J. Buceta, and F. J. de la Rubia, Physica A 224, 153 (1996).

[47] J. C. Micheau, W. Horsthemke, and R. Lefever, J. Chem. Phys. 81, 2450 (1984).

[48] P. de Kepper and W. Horsthemke, in Synergetics, Far From Equilibrium (Springer, New York, 1979).

[49] S. Kai, T. Kai, and M. Takata, J. Phys. Soc. Jpn. 47, 1379 (1979).

[50] M. Wu and C. D. Andereck, Phys. Rev. Lett. 65, 591 (1990).

[51] C. W. Meyer, G. Ahlers, and D. S. Cannell, Phys. Rev. 44, 2514 (1991).

[52] J. Martín-Regalado, S. Balle, and N. B. Abraham, IEEE J. Quantum Electron. 32, 257 (1996).

[53] H. K. Janssen, Z. Phys. B 42, 151 (1981); P. Grassberger, ibid. 47, 365 (1981); R. Dickman, Phys. Rev. E 50, 4404 (1994).

[54] T. Vicsek, in Proceedings of the Workshop on Surface Disordering: Growth, Roughening, and Phase Transitions, edited by R. Jullien, J. Kertesz, P. Meakin, and D. E. Wolf, Les Houches Series (Nova, New York, 1992) p. 155; A. L. Barabasi and H. E. Stanley, Fractal Concepts in Surface Growth (Cambridge University Press, Cambridge, 1995).

[55] P. E. Kloeden and E. Platen, Numerical Solution of Stochastic Differential Equations (Springer-Verlag, Berlin, 1992).

[56] R. Toral, in Third Granada Lectures in Computational Physics, edited by P. L. Garrido and J. Marro, Lecture Notes in Physics Vol. 448 (Springer, New York, 1995).

[57] R. Toral and A. Chakrabarti, Comput. Phys. Commun. 74, 327 (1993). 